\title{
Prototipo IaaS para el estudio del almacenamiento en bloque basado en Cinder
}

\section{IaaS prototype for the study of block storage based on Cinder}

\author{
GALLARDO-ALVAREZ, Dennise Ivonne $\dagger^{*}$, RAZÓN-GONZÁLEZ, Juan Pablo, DURAN-BELMAN, \\ Israel y MAGDALENO-ZAVALA, Juan Antonio
}

Instituto Tecnológico Superior de Irapuato, Carretera Irapuato - Silao km 12.5, Col. El Copal, C. P. 36821, Irapuato, Gto.

ID $1^{\mathrm{er}}$ Autor: Dennise Ivonne, Gallardo-Alvarez / ORC ID: 0000-0002-9197-6425, Researcher ID Thomson: S-4921-2018, CVU CONACYT ID: 264516

ID $1^{\text {er }}$ Coautor: Juan Pablo, Razón-González / ORC ID: 0000-0002-9457-5029, Researcher ID Thomson: S-4919-2018, CVU-CONACYT ID: 216902

ID $2^{\text {do }}$ Coautor: Israel Durán-Belman / ORC ID: 0000-0002-1394-0486, Researcher ID Thomson: V-7806-2019, CVU CONACYT ID: 691483

ID $3^{\text {er }}$ Coautor: Juan Antonio Magdaleno Zavala / ORC ID: 0000-0001-9762-0086, Researcher ID Thomson: S-5543-2018, CVU CONACYT ID: 691690

DOI: $10.35429 / J C E .2019 .7 .3 .21 .26$

Recibido 03 de Enero, 2019; Aceptado 30 Marzo, 2019

\begin{abstract}
Resumen
La evolución de la tecnología hacia una nueva era de flujos de trabajo ágiles y dinámicos en el tema de cómputo en la nube, ha dado lugar a que los profesionistas en Tecnologías de Información profundicen en el estudio de herramientas o mecanismos que optimicen el almacenamiento y la seguridad de los datos; por otro lado, para las empresas que manejan cada vez más un mayor volumen de datos para desarrollar su actividad, disponer de estas herramientas se ha convertido hoy en día en un factor clave. En este trabajo se da a conocer el estudio del componente Cinder de la plataforma OpenStack, como herramienta de almacenamiento en bloque que se asocia a máquinas virtuales o instancias; se presenta el despliegue de una plataforma de nube del modelo IaaS (Infraestructura como Servicio) en un entorno de pruebas, siguiendo las primeras cuatro fases de la metodología PDIOO propuesta por Cisco Systems; y se presentan los resultados que demuestran el almacenamiento persistente de la información a través del uso de dicho componente.
\end{abstract}

Infraestructura como Servicio, Almacenamiento, Cinder

\begin{abstract}
The evolution of technology towards a new era of agile and dynamic workflows in the topic of cloud computing has resulted in professionals in Information Technology deepening in the study of tools or mechanisms that optimize the storage and data security; on the other hand, for companies that increasingly handle a larger volume of data to develop their activity, having these tools has become a key factor today. In this work is present the study of the Cinder component of the OpenStack platform, as a block storage tool that is associated with virtual machines or instances; is presented the deployment of a cloud platform of the IaaS model (Infrastructure as a Service) in a test environment, following the first four phases of the PDIOO methodology proposed by Cisco Systems; and the results that demonstrate the persistent storage of information through the use of said component are presented.
\end{abstract}

Infrastructure as a Service, Storage, Cinder

Citación: GALLARDO-ALVAREZ, Dennise Ivonne, RAZÓN-GONZÁLEZ, Juan Pablo, DURAN-BELMAN, Israel y MAGDALENO-ZAVALA, Juan Antonio. Prototipo IaaS para el estudio del almacenamiento en bloque basado en Cinder. Revista de Ingeniería Civil. 2019. 3-7: 21-26

\footnotetext{
* Correspondencia del Autor (correo electrónico: degallardo@itesi.edu.mx)

$\dagger$ Investigador contribuyendo como primer autor.
} 


\section{Introducción}

En términos de Tecnologías de Información, la definición general del almacenamiento de datos suele ser la acción de guardar documentos o información en formatos ópticos o electromagnéticos en una computadora, no obstante, esta acción dentro de las empresas implica una mayor responsabilidad debido al valor de lo que se almacena (Evaluando Cloud, 2017). Hoy en día, las empresas se han encontrado con la necesidad de mejorar los mecanismos de almacenamiento a causa del incremento del número de datos producidos y los retos que surgen por el manejo de información.

\section{Existen distintos tipos de} almacenamiento disponible con diferentes características de uso, pero con cualquier sistema de almacenamiento las organizaciones deben tener en cuenta la regla 3-2-1 para que la disponibilidad de datos sea correcta; el nombre de esta regla hace referencia a la cantidad de elementos que la componen: Tres: Se refiere a la cantidad mínima de copias de información que se deben tener. Dos: Es la cantidad de medios diferentes en donde se deben realizar las copias. Uno: Una de las copias de seguridad debe encontrarse en un lugar fuera de las instalaciones (Pérez, 2019).

Desde una perspectiva más específica como el cómputo en la nube, existen tres tipos de almacenamiento de datos: a) Almacenamiento de objetos, b) Almacenamiento de archivos y c) Almacenamiento en bloque (Amazon Web Services, Inc., 2019).

El almacenamiento en bloque, que es el tema de estudio de este trabajo, es un modelo en donde los datos están alojados en espacios de almacenamiento virtualizados denominados "volúmenes" o "bloques" (TechTarget, 2017). Cada volumen actúa como un disco duro individual que se puede particionar, formatear y montar si así se requiere.

Mientras que hay muchas opciones en cuanto a un orquestador de nube, OpenStack es una opción popular del tipo IaaS en el mundo del código abierto; es una solución que controla grandes grupos de recursos de cómputo, almacenamiento y red, todo administrado a través de un panel de control (ver figura 1) que brinda a los administradores control, mientras permite a los usuarios proveerse de recursos a través de una interfaz web (OpenStack, 2019).
Dentro de los componentes de almacenamiento de OpenStack se encuentra "Cinder", el servicio de almacenamiento en bloque que está diseñado para presentar recursos de almacenamiento a usuarios finales (OpenStack, 2019). Este tipo de almacenamiento tiene grandes ventajas cuando se trabaja con virtualización de sistemas operativos en la nube ya que la información almacenada en cualquier volumen creado, asociado y des asociado a una instancia es persistente, esto aún después de que las instancias sean dañadas.

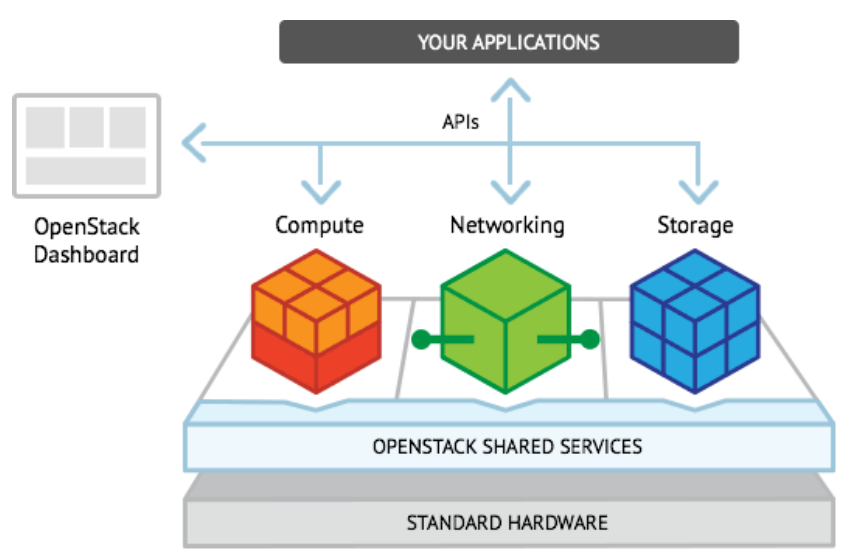

Figura 1 Metodología PDIOO

Fuente: (Mirantis, Inc., 2019)

En particular, dentro de las instituciones educativas que impartan formación relacionada con las TI, con Cinder se pueden aprovechar muchas de las ventajas del almacenamiento, ya que se pueden realizar respaldos dentro de los volúmenes para asociar a máquinas virtuales que permitan desplegar un nuevo escenario de trabajo en cuestión de minutos que en anteriores años tomaría días e incluso semanas en desplegar.

Ante este escenario y considerando lo descrito anteriormente, en este trabajo se plasma el análisis del comportamiento del componente Cinder de OpenStack, a través del desarrollo de un prototipo del modelo IaaS que permita la creación, asociación y desasociación de volúmenes de almacenamiento a instancias, a fin de visualizar las ventajas y/o desventajas para implementarlo como herramienta de almacenamiento a través de una nube privada institucional para las escuelas de nivel superior.

A lo largo del trabajo se presentan cada una de las fases de la metodología seguida en este proyecto, y se describen los resultados obtenidos así como las conclusiones a las que se ha llegado después de la culminación del estudio.

GALLARDO-ALVAREZ, Dennise Ivonne, RAZÓN-GONZÁLEZ, Juan Pablo, DURAN-BELMAN, Israel y MAGDALENO-ZAVALA, Juan Antonio. Prototipo IaaS para el estudio del almacenamiento en bloque basado en Cinder. Revista de Ingeniería Civil. 2019 


\section{Materiales y Métodos}

La metodología PDIOO (ver figura 2) seguida en este proyecto es propuesta por Cisco Systems y permite formalizar el diseño de una red en cinco fases: Planeación, Diseño, Implementación, Operación y Optimización (Cisco Systems, 2004).

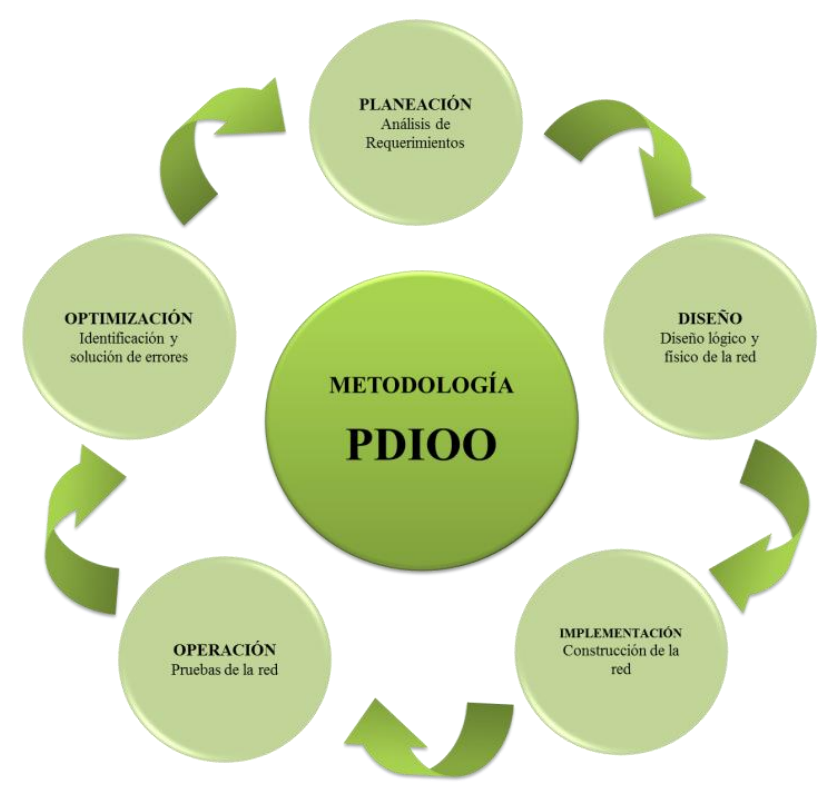

Figura 2 Metodología PDIOO

Fuente: Propia, basada en metodología de Cisco

\section{Planeación}

La planeación de una red, requiere la consideración de cada componente a utilizar. En la tabla 1 se describe tanto el hardware como el software utilizado para el desarrollo del entorno de pruebas:

\begin{tabular}{|c|c|c|c|}
\hline \multicolumn{4}{|c|}{ Hardware } \\
\hline & RAM & Procesador & $\begin{array}{l}\text { Disco } \\
\text { Duro }\end{array}$ \\
\hline $\begin{array}{l}\text { Computadora con } \\
\text { rol de Servidor }\end{array}$ & $\begin{array}{l}16 \\
\text { GB }\end{array}$ & $\begin{array}{l}\text { Intel Core i5 a } \\
2.3 \mathrm{GHz}\end{array}$ & $\begin{array}{l}250 \\
\text { GB }\end{array}$ \\
\hline $\begin{array}{l}\text { Computadora con } \\
\text { rol de cliente }\end{array}$ & $4 \mathrm{~GB}$ & $\begin{array}{l}\text { Intel Core i5 a } \\
1.7 \mathrm{GHz}\end{array}$ & $\begin{array}{l}250 \\
\text { GB }\end{array}$ \\
\hline \multicolumn{4}{|c|}{ Software } \\
\hline $\begin{array}{l}\text { Sistema Operativo } \\
\text { base }\end{array}$ & \multicolumn{3}{|c|}{ CentOS 7} \\
\hline $\begin{array}{ll}\begin{array}{l}\text { Software } \\
\text { virtualización }\end{array} & \text { de } \\
\end{array}$ & \multicolumn{3}{|c|}{ Virtual Box 6.0} \\
\hline Plataforma IaaS & \multicolumn{3}{|c|}{ OpenStack versión Ocata } \\
\hline $\begin{array}{l}\text { Sistema Operativo } \\
\text { Instanciado }\end{array}$ & \multicolumn{3}{|c|}{ Ubuntu 16.04 LTS } \\
\hline
\end{tabular}

Tabla 1 Hardware y Software utilizado en prototipo IaaS Fuente: Elaboración Propia
El sistema operativo base utilizado fue la versión mínima, es decir sin entorno gráfico, esto con la intención de ahorrar recursos en la computadora con el rol de servidor y tener un mejor rendimiento en el despliegue de la plataforma IaaS.

\section{Diseño}

Esta fase incluye el diagrama de red o la topología a implementar. Gracias a la virtualización basada en host y dado que el objetivo del proyecto se basa en el desarrollo de un prototipo, la instalación del entorno completo de OpenStack fue sobre una topología monolítica (una sola PC) y para las pruebas planteadas se diseñó una red LAN como se muestra en la figura 3.

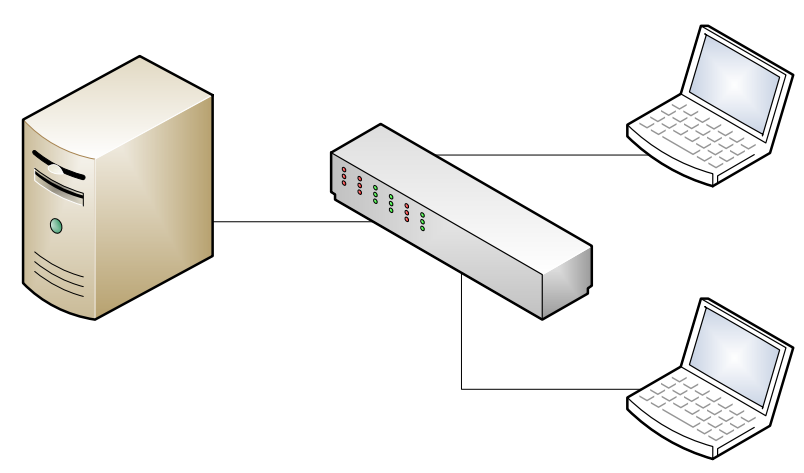

Figura 3 Topología de red

Fuente: Elaboración Propia

\section{Implementación}

Esta fase se centra en la preparación del entorno. En primer lugar se instaló el software de virtualización basado en host "VirtualBox" para la creación de una máquina virtual con el sistema operativo CentOS; sobre este sistema se instaló OpenStack-packstack versión Ocata y se configuraron cada uno de los componentes necesarios para la creación de instancias, además del componente Cinder para la creación, asociación y desasociación de volúmenes. Es importante documentar cada paso en la implementación, para regresar a escenarios anteriores en caso de fallas.

\section{Operación}

Esta fase incluye la gestión y el monitoreo de los componentes de la red. Se realizaron diversas pruebas que pueden resumirse en la creación de instancias, creación de volúmenes y asociación y desasociación de volúmenes a instancias. 


\section{Optimización}

Dado que OpenStack se instaló sobre una infraestructura monolítica donde el principal objetivo es comenzar a familiarizarse con la plataforma (específicamente el componente Cinder), su configuración y gestión, el alcance de este proyecto no cubre la fase de optimización pues no se llevó a un nivel de producción e interacción con el usuario; sin embargo, para trabajos posteriores y un mejor rendimiento, se recomienda una computadora con mayor capacidad en cuanto a hardware para evitar la alta latencia en los recursos virtualizados.

\section{Resultados}

La evaluación del funcionamiento del componente Cinder y la comprobación de la integridad de los datos almacenados en bloques se efectuaron a través de diversas pruebas que se centraron en la eliminación intencional de instancias con volúmenes asociados.

En la figura 4 se muestran las instancias creadas para la realización de pruebas, estas son del Sistema Operativo Ubuntu 16.04 LTS en modo consola, esto debido a las limitantes de hardware de la computadora con rol de servidor.

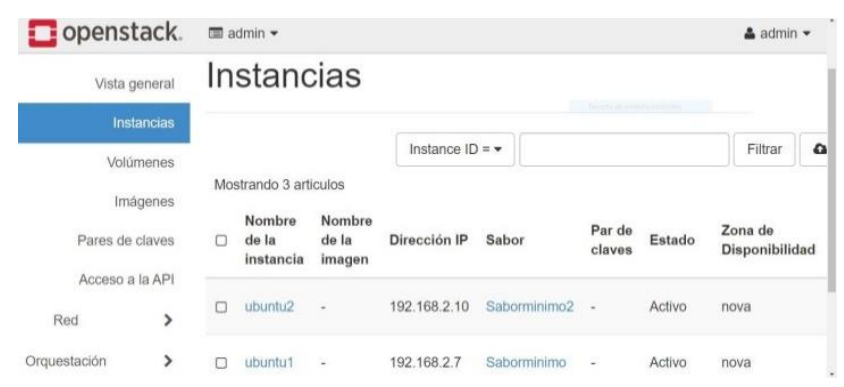

Figura 4 Instancias de prueba

Fuente: Elaboración Propia

Cuando se lanza una instancia, automáticamente se genera un volumen y se asocia a la misma, es decir, se crea su disco duro o su directorio de almacenamiento raíz. Los directorios raíz de cada instancia, son volúmenes arrancables ya que es donde se almacena en sí el sistema operativo instanciado pero dichos discos duros son efímeros: el contenido es temporal y dura el tiempo de vida de la instancia; cuando se termina la instancia la información guardada en ese disco duro, desaparece.
Para comprobar el almacenamiento en bloque persistente que ofrece Cinder, se creó un volumen de prueba (volumenTEST) que se asoció a las instancias como un segundo espacio de almacenamiento. Como se puede observar en la figura 5, el volumen creado y asociado manualmente a la instancia uno, no es arrancable ya que actúa simplemente como un disco duro adicional.

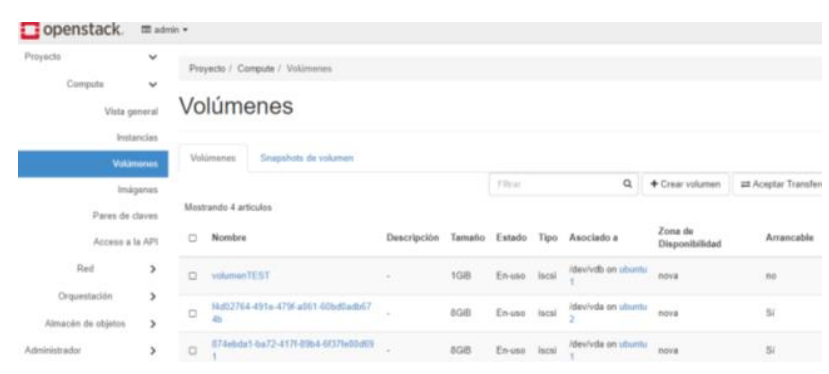

Figura 5 Volúmenes asociados, vista desde el dashboard de OpenStack

Fuente: Elaboración Propia

En la figura 6, se muestra la asociación de los mismos volúmenes de la imagen anterior pero ahora a través de la terminal de la instancia en ejecución desde el cliente.

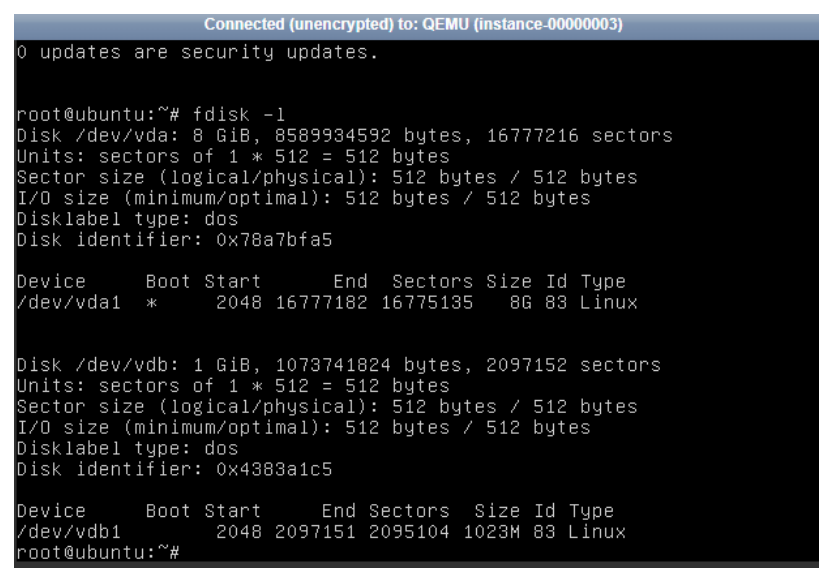

Figura 6 Volúmenes asociados a instancia uno Fuente: Elaboracion Propia

Para distinguir el disco duro raíz del volumen creado y asociado a la instancia, se puede observar la nomenclatura dada por el propio sistema operativo, donde $v d a$ es la unidad de almacenamiento raíz y $v d b$ es el espacio creado y asociado manualmente.

En la figura 7 se muestra el volumen $v d b$ montado en un directorio temporal y los archivos de prueba creados. 


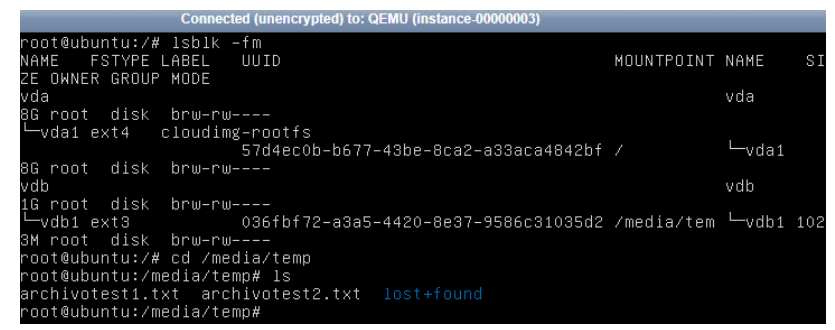

Figura 7 Archivos almacenados en vdb Fuente: Elaboración Propia

Por otro lado, una vez desasociado "volumenTEST" y asociado ahora a la instancia dos (ver figura 8), se pudo comprobar el almacenamiento permanente e independiente a la vida de una instancia.

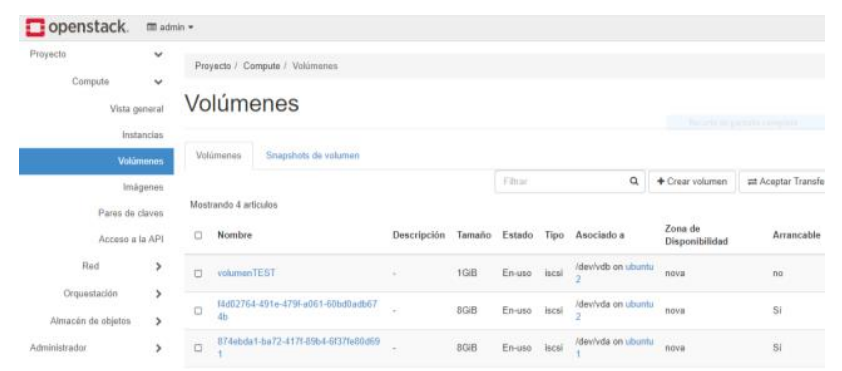

Figura 8 Volumen asociado a instancia dos Fuente: Elaboración Propia

De igual manera, en la figura 9 se aprecian dichos volúmenes a través de la terminal de la instancia dos en ejecución.

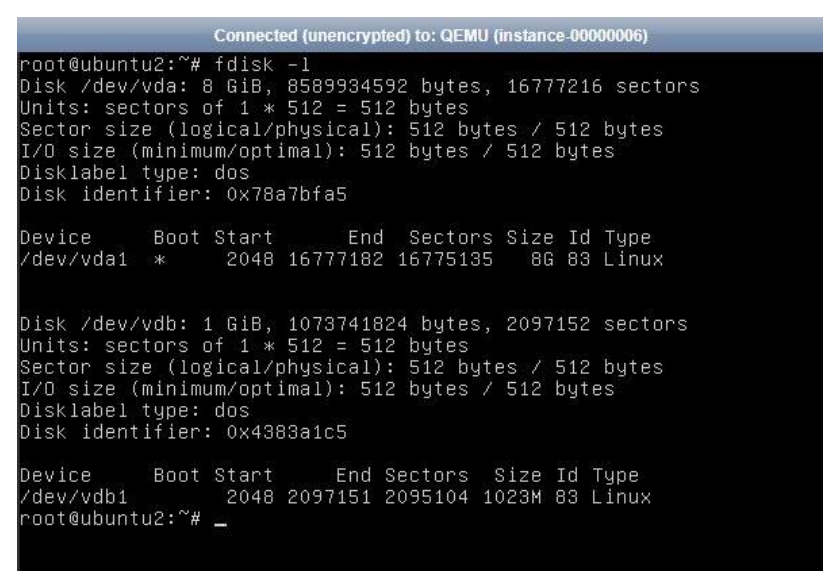

Figura 9 Volúmenes asociados a instancia dos Fuente: Elaboración Propia

$\mathrm{Y}$ como en el caso anterior, una vez montado el volumen, en la figura 10 se muestran los archivos de manera íntegra; creados y almacenados desde la primera asociación con la instancia uno.

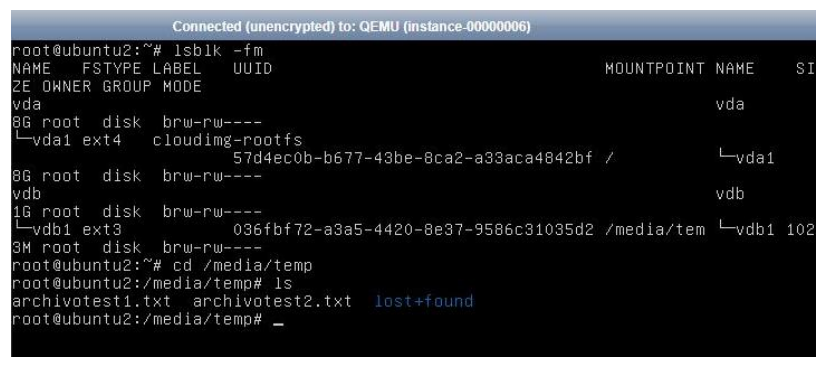

Figura 10 Archivos íntegros, almacenados en volumenTEST

Fuente: Elaboración Propia

Dados los resultados de estas pruebas es recomendable desmontar el volumen antes de desasociarlo, esto para evitar que el sistema de archivos se corrompa. Además, de acuerdo a las pruebas y a la investigación realizada, se encontró que hasta ahora no es posible asociar un volumen a más de una instancia a la vez, ni redimensionar el tamaño del volumen mientras esté asociado a una instancia.

Respecto a la eliminación de instancias con volúmenes asociados, se pudo comprobar que la información también se mantiene íntegra. En la figura 11 se muestra la eliminación de la instancia dos.

Instancias

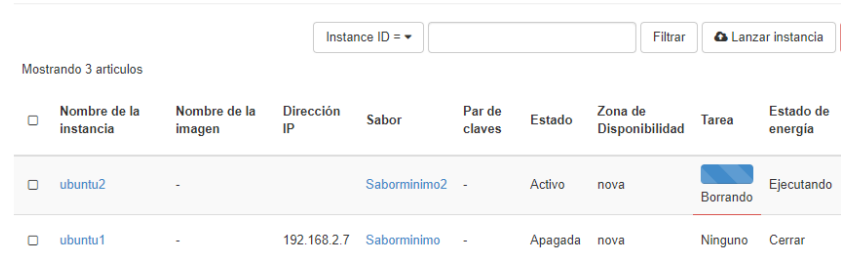

Figura 11 Eliminación de instancia dos Fuente: Elaboración Propia

Cuando la instancia se ha eliminado, los volúmenes que tenía asociados (tanto el directorio raíz como volumenTEST) se liberan automáticamente y quedan disponibles para ser asociados a alguna otra instancia (ver figura 12).

\section{Volúmenes}

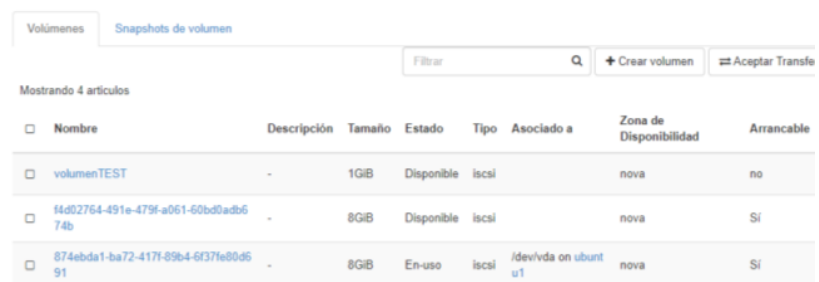

Figura 12 Volumen disponible

Fuente: Elaboración Propia 
Finalmente, en la figura 13 se muestran los dos volúmenes liberados por la instancia eliminada, asociados a la instancia uno.

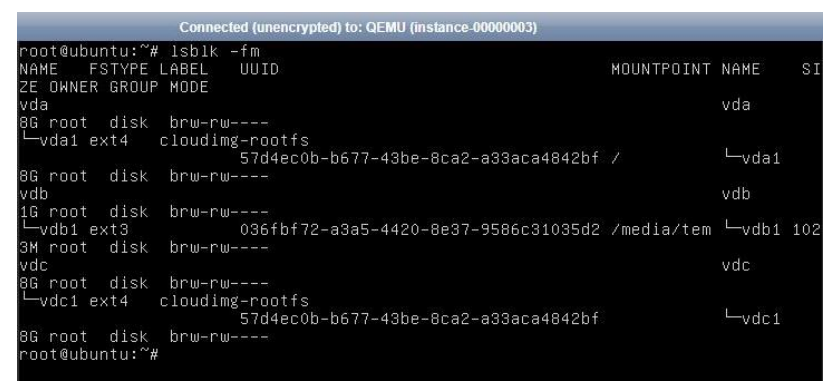

Figura 13 volúmenes liberados, asociados a instancia uno Fuente: Elaboración Propia

\section{Conclusiones}

Hoy en día se habla mucho de cómputo en la nube y transformación digital; OpenStack es una tecnología clave en dicha transformación, ya que aporta las herramientas necesarias para hacer frente de una forma rápida y ágil, a las exigencias a las que se enfrentan las organizaciones.

Los resultados esperados de este proyecto se cumplieron en un $100 \%$. Se logró desarrollar el prototipo obteniendo un sistema cloud funcional; OpenStack instalado y en operación. Se ha profundizado en su funcionamiento, arquitectura y el potencial que tiene el componente Cinder.

Es importante enfatizar que al hacer uso del cómputo en la nube, el hardware disponible en los equipos cliente no es tan relevante en términos de rendimiento, excepto en el caso del procesador y la tarjeta de red, por ello, para obtener mayor provecho de la IaaS lo recomendable para los equipos clientes es usar clientes livianos o ligeros.

Finalmente, el prototipo fue desarrollado en una computadora portátil de características óptimas para examinar el funcionamiento de la tecnología en un entorno de pruebas, pero para el despliegue de una nube institucional es necesario que se cuente con el hardware adecuado para la implementación de Cinder que soporte múltiples instancias con variedad de sistemas operativos y volúmenes, además de poder implementar otros servicios como virtualización de redes.

\section{Referencias}

Amazon Web Services, Inc. (2019). ¿Qué es el almacenamiento en la nube? | Backup en la nube AWS. Obtenido de https://aws.amazon.com/es/what-is-cloudstorage/

Cisco Systems, I. (2004). CCDA Self-Study: Designing for Cisco Internetwork Solutions (DESGN). (D. Teare, Ed.) Cisco Press.

Evaluando Cloud. (2017). Clasificación del almacenamiento de datos o Data Storage. Obtenido de https://evaluandocloud.com/clasificacion-delalmacenamiento-datos-data-storage/

Mirantis, Inc. (2019). OpenStack Overview. Obtenido de https://www.mirantis.com/openstack-/

OpenStack. (2019). Cinder - OpenStack. Obtenido de https://wiki.openstack.org/wiki/Cinder

OpenStack. (2019). Open source software for creating private and public clouds. Obtenido de https://www.openstack.org/

Pérez, A. (Mayo de 2019). Backup 3-2-1 para respaldar mi información. Obtenido de https://s4it.com.mx/wp/index.php/2019/05/10/b ackup-321/

TechTarget. (2017). ¿Qué es Almacenamiento de bloques? Obtenido de https://searchdatacenter.techtarget.com/es/defin icion/Almacenamiento-de-bloques 